\title{
Research on the System of Competitive Sports Based on Virtual Reality Technology
}

\author{
Yonggan Wang \\ Institute of Physical Education, Langfang Teacher's College, Langfang, China \\ w_angyonggan@126.com
}

Keywords: virtual reality technology; motion capture; articulation points; kinematic model; dynamic simulation

\begin{abstract}
The development of information technology has created increasingly sophisticated virtual reality technology. It enables efficient integration of computer hardware, software and virtual reality technology, and dynamic simulation of the real world. According to human action, language and others, dynamic environmental technology can timely respond to the state of athletes for real-time analysis, compensate for the randomness of tradition physical training plan. Based on motion capture technology of virtual reality, you can capture and analyze the articulation points to athletes. Establish kinematic model, to provide visual conditions for the dynamic simulation of competitive sports in sports training, achieve competitive sports system of technology innovation breakthroughs, stimulate the development of competitive sports technology update, and thus promoting the vigorous development of China's sports undertakings.
\end{abstract}

\section{Introduction}

Modern competitive sports is getting the rapid development with the characteristics of "high, hard, precise, and sharp”. Especially many people focused on sports investment in China, which has been used in the development of virtual reality technology, such as large-scale sports events, as the analysis of competitive sports program in the Olympic Games[1]. This makes sports training needs more with the help of modern means of science and technology. In order to maximize human potential, modern sports need science and technology constantly auxiliary. It requires kinematicsrelated knowledge, to discover the inherent regulation of sports with more systematic and scientific view[2]. Study of competitive sports based on virtual reality technology is an experimental science. It adopts to the computer stimulation movement and reproduce the teaching experience and intention of coach. It makes the manager's plan implement to each athlete's training course. And it can enable the explanation of sports system, analysis and science system of forecasting, planning and organization. In recent years, virtual reality technology application in sports has intellectualized simulation, qualitative and quantitative simulation, distributed interactive simulation, visual simulation, multi-media simulation and so on. The simulation is based on virtual reality technology, which focuses on a variety of perception, interaction and immersion. As the physical training requires athletes' variety of feeling (auditory, tactile, olfactory, visual) participatory role, simulation based on virtual reality technology will surely be in-depth mining application in sports field.

\section{Virtual reality technology}

Virtual reality technology(VR) consists of three basic characteristics, which are immersion, interaction and visualization. Immersion refers to the user as the man-machine environment exists in the virtual sense of the environment, is a state of mind[3]. Interaction is achieved by a combination between the virtual reality system and traditional three-dimensional animation. Visualization is that users get the perceptual and rational understanding from the qualitative and quantitative diagnosis of overall situation, deepen the concept of perception and germination. The virtual reality technology system is composed of professional image processing computer, utility software systems, enter the unit and presentation equipment. Multimedia is used to show the users' idea, but it can be only the one-way information from creator to audience: predetermined viewpoint, scheduled dialog box and 
the scheduled event results[4]. Although the audience can interact with the virtual world for simple interaction, it is only limited in two-dimensional, and lack of autonomy.

\section{The algorithm analysis of kinematics modeling}

Based on the kinematic modeling process and the object motion,we want to establish a process simulation modeling to changes the relative position, velocity and acceleration of the movement.It requires the use of forward kinematics, inverse kinematics and motion capture technology. Forward kinematics is the basis of the theory of setting key joint rotation angle and it obtains a corresponding relation of each limb position information. The inverse kinematics theory is based on specifying the players' terminal joint position. And then the computer automatically calculate and simulate various intermediate joint relative position. The following will mainly introduce rate method in the Jacobi matrix method, which can deal with movement simulation process more complex case by virtual reality technology.Sports simulation can capture motions by optical or electromagnetic means[5]. Through capturing the multiple motion clips unite of moving point, it seamless connects the end of last actions and the starting of next action.

Through the Jacobi matrix, it can be calculated from the joint chain of each joint from the freedom to the change of the volume. According to the virtual work principle of Jacobi transpose method, end effector in joint position changes the force, then changes the joint angles. By using the Jacobi matrix method for joint chain, joint degrees of freedom is is an iterative process that should be solved.

Jacobi algorithm matrix formula is as follows[6]:

$$
\left\{\begin{array}{c}
x=f(\theta) \\
\Delta x=J(\theta) \Delta \theta \\
\Delta \theta=J^{-1} \Delta x
\end{array}\right.
$$

wherein, $J(\theta)$ is expressed as a function of a $6 \times n$ Jacobi matrix about function $f, \mathrm{n}$ is for joint number, $\theta$ is for the freedom degree of each chain joint, and the relative position is $\mathrm{X}$.

\section{Empirical analysis on dash project based on the Jacobi matrix method}

The athlete's body can be seen as a joint tree structure. A joint tree consists of a lot of branches to form a number of joint chains. A joint chain is connected by joints through bones[7]. The end of the joint is generally referred to as end-effectors. The vector $\theta=\left(\theta_{1}, \theta_{2}, . ., \theta_{n}\right)$ expresses the degree of freedom of each joint on joint chains, the position of the end-effectors $\mathrm{x}$ can only be sure by $x=f(\theta)$. If the position of the end-effectors $\mathrm{x}$ is known, it can also reverse to know the degree of freedom $\theta$ of each joint on joint chain. The following mainly introduces the Jacobi matrix, CCD and DLS.

To derive $x=f(\theta), \Delta x=J(\theta) \Delta \theta$ can be deduced by the vector function differential calculus. Wherein $J(\theta)$ is $6 \times n$ which is Jacobi matrix about function $\mathrm{f}, \mathrm{n}$ is a number of joints. Then it derives the degrees of freedom variation from each joint on joint chains, namely $\Delta \theta=J^{-1} \Delta x$. Jacobi transposition method regards the position change of the end-effectors as force, according to the principle of virtual work. And also it regards the change of the joints' angle as force, then solves by $\Delta \theta=J^{T} \Delta x$ [8]. The following figure shows the block diagram of the degree of freedom of each joint on joint chains by using Jacobi transposition method. The solving process is an iterative process.

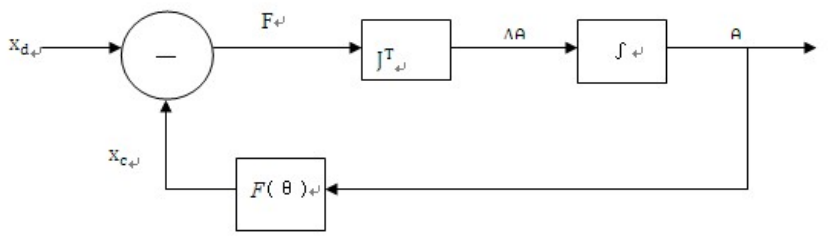

Figure 1. The block diagram by Jacobi matrix transposition method 
It can access to i-th joint by using Jacobi matrix computing method of ${ }^{J_{i}}$. Set ${ }^{U_{i}}$ as the rotary spindle of i joint, ${ }^{M_{i}}$ as rotation matrix of i joint, transforming i joint from local coordinate system to the world coordinate system, axis $_{i}$ is the result from the transformation matrix, namely axis $_{i}=u_{i} M_{i}$. Because the joints in the human body's joint chains is rotating without moving, so there is a result $J_{i}=\left[\begin{array}{c}{\left[\left(x-j_{i}\right) \times \text { axis }_{j}\right]^{T}} \\ {\left[\text { axis }_{j}\right]^{T}}\end{array}\right]$. The $\mathrm{x}$ in the equation represents the position of the endeffectors in the coordinates, ${ }^{j}{ }_{i}$ represents the position of $i$ in the coordinates[9].

Cyclic coordinate descent method is called CCD method. It is based on an optimization method, the basic idea is to convert the $\theta=f^{-1}(x)$ to a minimization problem, namely $\min E(\theta)=(P-f(\theta))^{2}$. Wherein, p represents the target position of the end. A variety of iterative method in nonlinear programming can solve the minimization problem. Generally $\theta$ is under control, and it can convert to a constrained nonlinear optimization problem, namely $\min E(\theta)=(p-f(\theta))^{2}$, s.t.l $l_{i} \leq \theta_{i} \leq u_{i}, i=1, . ., n$. CCD method avoids numerical iterative method of Jacobi matrix and its inverse solution, thus the speed has been greatly improved. It starts from the end of the articulated chain to the root direction to adjust the angle of each joint in proper order. Each adjustment of the terminal is approximating to the position of target. The progress is iterative. Guiding terminal and the distance of the position of target are within a certain error range, then, it is a successful solution.

DLS method is also called damped least-squares. When you deal with a single end-effectors situation, you can use Jacobi transposition method or CCD method to achieve satisfactory accuracy and real-time. But dealing with multiple end-effectors, using the methods what has been said before is not only low convergence, but also the target location can not be reached, and even there is a oscillation. So, DLS method is more available.

According to the above analysis,End position ( $\mathrm{x}, \mathrm{y}$ ) and the joints' displacement can be obtained by the following kinematic equation[10]:

$$
\left\{\begin{array}{l}
x=l_{1} \cos \theta_{1}+l_{2} \cos \left(\theta_{1}+\theta_{2}\right) \\
y=l_{1} \sin \theta_{1}+l_{2} \sin \left(\theta_{1}+\theta_{2}\right)
\end{array},\right.
$$

We can reverse the launch of the joint position and the angle of the joint.

$$
\left\{\begin{array}{l}
d x=\frac{\partial x}{\partial \theta_{1}} d \theta_{1}+\frac{\partial x}{\partial \theta_{2}} d \theta_{2} \\
d y=\frac{\partial y}{\partial \theta_{1}} d \theta_{1}+\frac{\partial y}{\partial \theta_{2}} d \theta_{2}
\end{array}\left[\begin{array}{l}
d x \\
d y
\end{array}\right]=\left[\begin{array}{cc}
\frac{\partial x}{\partial \theta_{1}} & \frac{\partial x}{\partial \theta_{2}} \\
\frac{\partial y}{\partial \theta_{1}} & \frac{\partial y}{\partial \theta_{2}}
\end{array}\right]\left[\begin{array}{l}
d \theta_{1} \\
d \theta_{2}
\end{array}\right]=\left[\begin{array}{cc}
-l_{1} s_{1}-l_{2} s_{12} & -l_{2} s_{12} \\
l_{1} c_{1}+l_{2} c_{12} & l_{2} c_{12}
\end{array}\right]\left[\begin{array}{l}
d \theta_{1} \\
d \vartheta_{2}
\end{array}\right],\right.
$$

From Figure 3,

$$
V=\left[\begin{array}{l}
v_{x} \\
v_{y}
\end{array}\right]_{=}\left[\begin{array}{cc}
-l_{1} s_{1}-l_{2} s_{12} & -l_{2} s_{12} \\
l_{1} c_{1}+l_{2} c_{12} & l_{2} c_{12}
\end{array}\right]\left[\begin{array}{l}
\varphi_{1} \\
\varphi_{2}
\end{array}\right],
$$

In which, ${ }^{\varphi}$ is for the joint's velocity, $\mathrm{V}$ for a generalized velocity $, \varphi_{1}, \varphi_{2}$ are the speed of joint point at one time, which can be standed for $\varphi=J^{-1} V$.

For Jacobi matrix method is reversible, we cant get the formula[11]:

$$
J^{-1}=\left[\frac{1}{l_{1} l_{2} s_{2}}\right]\left[\begin{array}{cc}
l_{2} c_{12} & l_{2} s_{12} \\
-l_{1} c_{1}-l_{2} c_{12} & -l_{1} s_{1}-l_{2} s_{12}
\end{array}\right],
$$

We can get the velocity of joint point[12]: 


$$
\begin{aligned}
& \varphi_{1}=\frac{l_{2} v_{x} \cos \left(\theta_{1}+\theta_{2}\right)+l_{1} v_{y} \sin \left(\theta_{1}+\theta_{2}\right)}{l_{1} l_{2} \sin \theta_{2}} \\
& \varphi_{2}=-\frac{v_{x}\left[l_{1} \cos \theta_{1}+l_{2} \cos \left(\theta_{1}+\theta_{2}\right)\right]+v_{y}\left[l_{1} \sin \theta_{1}+l_{2} \sin \left(\theta_{1}+\theta_{2}\right)\right]}{l_{1} l_{2} \sin \theta_{2}}
\end{aligned}
$$

By above knowable, sprint A knee joint B C hip shoulder D wrist E ankle as the main point of articulation, the state of motion can be divided into: touchdown early, through in the knee and hip joint torque ( EXF ) forward action, then the muscle torque ( MUS ) is the main resistance the ground reaction force; stretch late in the hip joints, there will be considerable thigh acceleration and hip acceleration, away from the shank after it starts to work. Two is the initial swing, the shank through the inertia torque to drive the thigh, the moment of inertia ( INT ) and muscle torque value to a maximum, then used in knee and hip; support phase, the outer torque ( EXF ) and muscle torque ( MUS ) at the same time, to the end of the acceleration of the thigh and hip acceleration force generation large torque so that the shank strength greater; three is the empty stage, the thigh crus is mainly by the control moment of inertia ( INT ) and muscle torque ( MUS ) control, complete the dash movement.

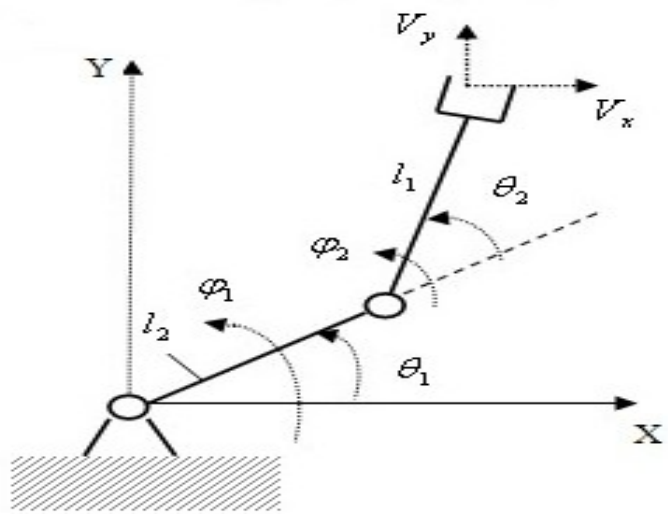

Figure 2. $\quad$ Dynamic analysis of sprint starting

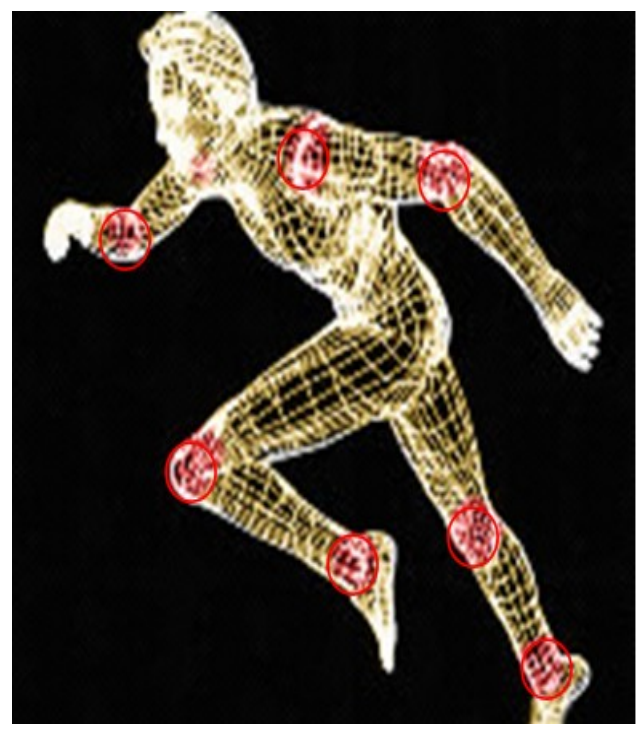

Figure 3.

The joint point diagram of infrared high speed photography

TABLE I. The generated torque of vertical ground reaction force list $( \pm \mathrm{SD})$

\begin{tabular}{|c|l|l|}
\hline Joint point & Muscle torque (Nm) & external torque(Nm) \\
\hline A knee joint & $203.40 \pm 93.60$ & $96.82 \pm 76.07$ \\
\hline B hip & $455.24 \pm 198.72$ & $218.58 \pm 130.99$ \\
\hline C shoulder joint & $303.40 \pm 87.60$ & $153.82 \pm 66.07$ \\
\hline D wrist joint & $478.24 \pm 198.72$ & $238.48 \pm 135.99$ \\
\hline E ankle & $179.30 \pm 63.50$ & $69.82 \pm 45.07$ \\
\hline
\end{tabular}

TABLE II. The acceleration peak list of terminal swing thigh, leg angular velocity and angular ( \pm SD )

\begin{tabular}{|c|l|l|}
\hline & angular velocity (rad/s) & angular acceleration $\left(\mathbf{r a d} / S^{2}\right)$ \\
\hline lower leg & $11.98 \pm 1.55$ & $400.36 \pm 70.72$ \\
\hline Thigh & $6.46 \pm 1.37$ & $52.93 \pm 36.05$ \\
\hline Upper arm & $10.67 \pm 1.47$ & $420.76 \pm 74.51$ \\
\hline Forearm & $7.56 \pm 1.41$ & $54.53 \pm 29.11$ \\
\hline palm & $4.46 \pm 0.72$ & $37.23 \pm 17.28$ \\
\hline
\end{tabular}

Through Table 1,Table 2 and Jacobi matrix method,the end effector force and torque were analyzed, concluding the influence factors of joint sprinter results: vacated early leg extends down the fast swing force.And in vacated stage,the speed peak is determined by the clockwise angular acceleration of the thigh.It is largest when speed is produced in order to reach maximum angle.Through the analysis, when the maximum energy of 41, velocity values of knee and hip joint can reach the maximum. 


\section{Conclusion}

Virtual reality technology has been widely used in competitive sports as a high-tech. According to the simulation of 3D motion information, it can draw up training plan, and to have a scientific assessment and reasonable choice by collecting various information, such as collecting the psychological and physiological index of athletes, training environment and simulating opponent. Therefore, a variety of scientific research project based on virtual reality technology has been paid more attention because of its remarkable effects. Virtual reality technology can be served in sports training. It is of great significance towards the development of competitive sports. The initial establishment of kinematics models, directly reflecting the real time state of athletes, inspiring athletes to challenge self-limits, promote the development of China's sports system.

\section{References}

[1] Wang Hongyu, Talking about the application of virtual reality technology in sports training . Journal of Jilin Institute of Physical Education, 2003, 23 (2):89-92.

[2] Wang Jie. Zhang Qingwen. Experimental teaching system construction is based on the study of training mode of sports information technology . Journal of Shanghai Institute of Physical Education,2012(3):49-55

[3] Wang Chengwei,Gao Wen. VR (virtual reality) technology theory, implementation and Applications.Tsinghua University press,2006 : 111-120

[4] Wang Jin, Ni Jianhong. Computer virtual reality technology in modern sports.Science of Surveying and Mapping, coal technology, 2011(6):67-69.

[5] Song Li. Discussion about application of virtual reality technology in sports simulation . Journal of Xi'an University of Posts and Telecommunications, 2007,12(6):54-57

[6] Li Xin. Virtual reality and its educational applications . Science Press. 2008(06):10-14

[7] Li Yangchen, Sun Jinhai. Sports simulation system.People's Sports Publishine House of China, 2001:178-184.

[8] Yu Gungyun, Li Xiaoping. The future of sports science technology - virtual reality technology . China sport Science and Technology, 2011,31(12) : 59.

[9] Chen Rong. Nonprofit and commercial operation of sports under the conditions of a market economy . Journal of Shanghai University of Sport, 2009,23(2) : 14-19.

[10] Su Jianming, Zhang Xuhong. The prospect of virtual reality technology.Computer emulation, 2011(2):18-21

[11] Chen Jian, Yao Songping. Applications of virtual reality technology in sports simulation . Sport Science ,2009(9):89-91.

[12] Gu Fuming.Research on Simulated Modeling Body Motion by Computer .Chengdu Sports University Journal, 2007,33(3):90-93. 